\title{
Auto-fluorescence spectra of oral submucous fibrosis
}

\author{
Hsin-Ming Chen ${ }^{1,2}$ \\ Chih-Yu Wang ${ }^{3}$ \\ Chin-Tin Chen ${ }^{4}$ \\ Hsiang Yang ${ }^{2}$ \\ Ying-Shiung Kuo ${ }^{1,2}$ \\ Wan-Hong Lan ${ }^{1,2}$ \\ Mark Yen-Ping Kuo ${ }^{1,2, *, \dagger}$ \\ Chun-Pin Chiang ${ }^{1,2, \dagger}$
}

\footnotetext{
${ }^{1}$ School of Dentistry, College of Medicine, ${ }^{2}$ Department of Dentistry, National Taiwan University Hospital, Taipei,

${ }^{3}$ Department of Biomedical Engineering, I-Shou University, Kaohsiung, Taiwan, and

${ }^{4}$ Center for Optoelectronic Biomedicine, College of Medicine, National Taiwan University, Taipei, Taiwan

${ }^{*}$ Co-corresponding author: Mark Yen-Ping Kuo, School of Dentistry, College of Medicine, National Taiwan University, 1 Chang-Te Street, Taipei, Taiwan. Tel.: +8862 23123456/ext. 7083; fax: +88622383 1346; e-mail: oddie@ccms.ntu.edu.tw.

${ }^{\dagger}$ These two authors have equally contributed in this paper.
}

\begin{abstract}
Background: Oral submucous fibrosis (OSF) is a chronic oral mucosal disease characterized by progressive deposition of collagen in the subepithelial connective tissue and epithelial atrophy. Previous studies have shown that at 330-nm excitation, the 380- and 460-nm emission peaks of the auto-fluorescence spectra for oral mucosal tissues reflect the collagen content in the subepithelial connective tissue and the nicotinamide adenine dinucleotide phosphate (NADH) content in the epithelial cells, respectively. Therefore, at 330-nm excitation OSF mucosa may have a higher 380-nm emission peak and a lower 460-nm emission peak than the normal oral mucosa (NOM).

Methods: To test the above hypothesis, we measured the in vivo auto-fluorescence spectra of 59 OSF mucosal sites and compared the measured spectra with auto-fluorescence spectra obtained from 15 NOM samples from 15 healthy volunteers, five samples of friction hyperkeratosis (histologic diagnosis, hyperkeratosis and acanthosis) on OSF buccal mucosa (FHOSF), and 29 samples of oral leukoplakia (histologic diagnosis, hyperkeratosis and acanthosis) on OSF buccal mucosa (OLOSF).

Results: We found that the spectrum of the OSF mucosa had a significantly higher 380-nm emission peak and a significantly lower 460-nm emission peak than the spectra of NOM, FHOSF, and OLOSF samples. When the mean $( \pm S D)$ fluorescence intensities at $380 \pm 15 \mathrm{~nm}\left(I_{380 \pm 15 \mathrm{~nm}}\right)$ and $460 \pm 15 \mathrm{~nm}\left(I_{460} \pm 15 \mathrm{~nm}\right)$ emission peaks and the mean ratio of $I_{460 \pm 15 \mathrm{~nm}} / I_{380 \pm 15 \mathrm{~nm}}$ were compared between groups, we found that OSF group had a significantly higher mean value of $I_{380 \pm 15 n m}$, a significantly lower mean value of $I_{460 \pm 15 \mathrm{~nm}}$, and a significantly lower mean ratio of $I_{460 \pm 15 \mathrm{~nm}} / I_{380 \pm 15 \mathrm{~nm}}$ than the NOM, FHOSF, and OLOSF groups $(P<0.001)$. However, no significant differences in the mean values of $I_{380 \pm 15 \mathrm{~nm}}, I_{460 \pm 15 \mathrm{~nm}}$, and ratio of $I_{460 \pm 15 \mathrm{~nm}} /$ $I_{380 \pm 15 \mathrm{~nm}}$ were found between NOM and FHOSF or OLOSF samples as well as between FHOSF and OLOSF samples ( $P>0.05)$.

Conclusion: Because OSF mucosa has a very unique pattern of auto-fluorescence spectrum, we conclude that auto-fluorescence spectroscopy is a good method for real-time diagnosis of OSF.
\end{abstract}

Key words: auto-fluorescence spectroscopy; oral submucous fibrosis

J Oral Pathol Med 2003: 32: 337-43

Oral submucous fibrosis (OSF) is a chronic scaring oral disease characterized by epithelial atrophy and progressive deposition of 
collagen in the lamina propria and submucosa of the oral mucosa. Epidemiologic studies have shown the intimate relationship between the areca quid (AQ) chewing habit and OSF (1-3). Our recent studies have also demonstrated that all the OSF patients in Taiwan have the AQ chewing habit (4-6). The areca nut alkaloids can stimulate the fibroblast proliferation and collagen synthesis (7). Cytokines and growth factors produced by inflammatory cells within the OSF tissues may promote fibrosis by inducing proliferation of fibroblasts, upregulating collagen synthesis, and downregulating collagenase production (8). The areca nut polyphenols, $(+)$-catechin and tannins, can stabilize the collagen structure $(9,10)$. OSF fibroblasts have been shown to produce the collagen with more stable structure such as collagen type I trimer (11) and secrete more lysyl oxidase, which causes an increase in collagen cross-linkages $(12,13)$. In addition, OSF fibroblasts secrete less amount of collagenase (14) and have less collagen phagocytosis activity than normal oral mucosal fibroblasts (15).

In Taiwan, there are two million people who have AQ chewing habit (16). The prevalence of OSF in AQ chewers in Taiwan has not been studied. However, an OSF incidence of $0.1-3.4 \%$ has been reported (17-21). In China, the prevalence of OSF is about $3 \%$ in a group of $\mathrm{AQ}$ chewers in Xiangtan City, Hunan Province (21). By a more conservative estimation of an OSF prevalence of $1 \%$, there may be about 20000 OSF patients in Taiwan. Although OSF can be diagnosed clinically, sometimes it may need a biopsy to confirm the clinical diagnosis. Therefore, it will be very convenient for the clinicians if there is a diagnostic technique that can make a real-time diagnosis of OSF without necessity of doing an incisional biopsy. Autofluorescence spectroscopy seems to have high potential to perform this function.

Auto-fluorescence spectroscopy has been shown to be a promising technique for distinguishing neoplastic from normal tissues in a variety of organs, including lung (22), breast (22), bronchus $(23)$, cervix $(24,25)$, colon $(26-28)$, urinary bladder (29), and head and neck (30-35). The principle of auto-fluorescence spectroscopy is based on the fact that, compared to normal tissues, different diseased tissues contain different morphohistologic characteristics and intrinsic fluorophores that give rise to different fluorescence emission spectra when the tissues are excited at a suitable wavelength $(36,37)$. Gillenwater et al. (33) showed that a diagnostic algorithm based on spectra at 337-nm excitation could discriminate normal from dysplastic and malignant oral mucosa with a sensitivity of $88 \%$ and a specificity of $100 \%$. Ingrams et al. (34) found that a diagnostic statistical algorithm based on the fluorescence measurements with 410-nm excitation light could differentiate histologically normal from dysplastic and cancerous oral tissues with the sensitivity, specificity, and overall accuracy being 90,91, and 91\%, respectively. Our previous ex vivo study demonstrated that at 330-nm excitation the spectra of human oral cancers had a lower 380-nm emission peak and a higher 460-nm emission peak than those of normal oral mucosa (NOM) samples (35). By use of 7, 12-dimethylbenz[a] anthracene (DMBA)-induced hamster buccal pouch carcinogenesis model, we also showed that at 330-nm excitation the intensity of the 380$\mathrm{nm}$ emission peak gradually decreased, while the intensity of the 460-nm emission peak gradually increased as the normal buccal pouch tissues transformed into dysplastic and further into cancerous tissues compared with the spectra of normal buccal pouch samples (35). All these findings indicate that auto-fluorescence spectroscopy can be used to differentiate normal from precancerous and cancerous oral tissues. However, no one has attempted to use auto-fluorescence spectroscopy to diagnose OSF lesions in vivo.

It has been demonstrated that, under the range of 300-360-nm excitation wavelength, the emission band at $380-400 \mathrm{~nm}$ is mainly attributed to the presence of collagen, while that at $440-460 \mathrm{~nm}$ is mainly due to the presence of nicotinamide adenine dinucleotide phosphate (NADH), as measured in both purified chromophores and tissue extracts $(25,26,38,39)$. Using $N$-nitrosomethyl benzylamine (NMBA)-induced rat esophageal carcinogenesis model, Glasgold et al. (39) further showed that the fluorophore responsible for the 390-nm emission peak exists in the subepithelial connective tissue (collagen) and that responsible for the 450-nm emission peak exists in the epithelium $(\mathrm{NADH})$. OSF mucosa has increased deposition of collagen in the subepithelial connective tissue and the atrophic oral epithelium. Therefore, at 330-nm excitation OSF mucosa may have a higher 380-nm emission peak and a lower 460-nm emission peak than the NOM. To test this hypothesis, we employed a fiber optics-based fluorescence spectroscopy system to measure the in vivo auto-fluorescence spectra of 59 OSF buccal mucosal sites and compared the measured spectra with auto-fluorescence spectra obtained from 15 NOM samples from 15 healthy volunteers, five samples of friction hyperkeratosis $(\mathrm{FH})$ on OSF buccal mucosa (FHOSF), and 29 samples of oral leukoplakia (OL) on OSF buccal mucosa (OLOSF). We found that the spectrum of the OSF mucosa had a significantly higher 380-nm emission peak and a significantly lower 460-nm emission peak than the spectra of NOM, FHOSF, and OLOSF samples. Because OSF mucosa has a very unique pattern of auto-fluorescence spectrum, we suggest that auto-fluorescence spectroscopy is a good method for realtime diagnosis of OSF. 


\section{Materials and methods}

Fifty-nine OSF patients (mean $\pm \mathrm{SD}=38.2 \pm 10.6$ years, range $=$ 18-62 years) were recruited from the Department of Oral and Maxillofacial Surgery, National Taiwan University Hospital (NTUH). Clinical diagnosis of OSF was made when patients showed characteristic features of OSF, including intolerance to spicy foods, blanching and stiffness of the oral mucosa, fibrous bands in the buccal or labial mucosa, and progressive inability to open the mouth. Some OSF patients might also have a burning sensation in the mouth, xerostomia, the presence of vesicles or ulcers on the oral mucosa, depapillation of the tongue, and impaired tongue mobility. The oral mucosal sites (soft palate, retromolar area, buccal mucosa, labial mucosa, floor of the mouth, and tongue) of involvement and the maximal mouth opening (MMO) of OSF patients were recorded. Clinical severity of OSF was determined according to the sites of involvement or MMO; the more the sites involved and the less the MMO, the more severe the OSF. No mild OSF case was included in this study, because all our OSF cases had at least three sites of involvement. Of the 59 OSF patients, five also had FHOSF and 29 also had OLOSF. FH was a white oral mucosal lesion caused by the chronic irritation from an elongated molar tooth or an ill-fitted dental prosthesis. OL is defined as a white patch or plaque that cannot be characterized clinically or pathologically as any other disease. After taking in vivo spectroscopic measurements, incisional or excisional biopsy was taken from all buccal mucosal sites of OSF, FHOSF, and OLOSF lesions where fluorescence spectra were taken. Clinical diagnosis was confirmed by histopathological examination of the biopsy specimens. The histological criteria for a diagnosis of OSF were: (i) atrophic epithelium with parakeratosis or hyperkeratosis, and (ii) moderate-to-marked fibrosis or hyalinization in the lamina propria, submucosa, and superficial muscle layer. All the FH and OL lesions on OSF buccal mucosa showed hyperkeratosis (hyperorthokeratosis or hyperparakeratosis) and acanthosis without epithelial dysplasia.

All the 59 OSF patients had AQ chewing habit; they chewed $3-100$ quids/day (mean $\pm \mathrm{SD}=27.6 \pm 22.0$ quids/day) for $1-38$ years $(14.0 \pm 9.2$ years). Fifty-five of the 59 (93.2\%) OSF patients were smokers; they smoked 2-60 cigarettes/day $(21.7 \pm 12.1$ cigarettes/day) for 3-40 years (17.8 \pm 9.3 years). Forty-seven of the 59 (79.7\%) OSF patients were alcohol drinkers; they drank $5.3-1645 \mathrm{~g}(380.1 \pm 456.0 \mathrm{~g})$ of pure alcohol/week for 0.5-40 years $(15.7 \pm 10.4$ years). Fifteen healthy patients (mean $\pm \mathrm{SD}=39.5 \pm$ 12.3 years, range $=22-60$ years) with dental caries only and without any oral mucosal diseases and oral habits were used as normal controls. Informed consent was obtained from each patient. This study was approved by the Ethic Committee on Use of Humans as Experimental Subjects of NTUH.

\section{Auto-fluorescence spectroscopy and statistical analysis}

In vivo fluorescence excitation-emission spectra were obtained from each OSF, FHOSF, or OLOSF lesion and each normal buccal mucosal site of the control patients using a handheld optical fiber probe attached to a spectrofluorometer (SkinSkan, JC Inc., Urbana, IL, USA). A monochromator with a $150-\mathrm{W}$ ozone-free Xenon lamp provides the excitation light. The desired excitation wavelength and the emission spectrum were selected by motorcontrolled monochromator. The excitation light was guided to illuminate samples by one arm of a Y-type quartz fiber bundle, and the emission fluorescence was collected by another arm of the fiber bundle. The optical fiber probe was disinfected with $2.4 \%$ glutaraldehyde solution, rinsed with phosphate buffered saline, and wrapped with transparent plastic membrane before each clinical use.

We have previously shown that the auto-fluorescence spectroscopy at 330-nm excitation could differentiate oral precancerous or cancerous lesions from NOM (35). Therefore, in this study 330-nm wavelength was used to excite each sample, and the resulting emission spectra were recorded from 340 to $615 \mathrm{~nm}$ in 3-nm increments. During measurement, the probe was gently touched on the surface of normal or lesional buccal mucosal site. The spectrum at each site was obtained by averaging three spectra measured from three adjacent sites ( $2 \mathrm{~mm}$ apart) of the same lesion.

To avoid the intensity variations due to different excitation light power and fluorescence collection efficiency, all spectra were normalized by dividing the intensity of each wavelength by the integrated area under the total emission spectrum. After normalization, the mean $( \pm \mathrm{SD})$ fluorescence intensities at $380 \pm$ $15 \mathrm{~nm}\left(I_{380 \pm 15 \mathrm{~nm}}\right)$ and $460 \pm 15 \mathrm{~nm}\left(I_{460 \pm 15 \mathrm{~nm}}\right)$ emission peaks and the mean ratio of $I_{460 \pm 15 \mathrm{~nm}} / I_{380 \pm 15 \mathrm{~nm}}$ were calculated for NOM, OSF, FHOSF, and OLOSF samples. The differences in the mean values of $I_{380 \pm 15 \mathrm{~nm}}, I_{460 \pm 15 \mathrm{~nm}}$, and ratio of $I_{460 \pm 15 \mathrm{~nm}} /$ $I_{380 \pm 15 \mathrm{~nm}}$ between any two of the NOM, OSF, FHOSF, and OLOSF groups were assessed for statistical significance by Mann-Whitney rank sum test. As the distributions of daily and total consumption of AQs, cigarettes and alcohol as well as duration of these oral habits do not follow normal distribution, these variables were treated as dichotomous variables by selecting the median value as the cutoff point. Similarly, the distribution of MMO does not obey normal distribution; instead of using 
interval scale a binary variable using the median as the cutoff point was adopted to express the clinical severity of OSF. The clinical severity of OSF judged by sites of involvement was subdivided into two groups: moderate OSF with three or four sites of involvement $(n=25)$ and severe OSF with five or six sites of involvement ( $n=34)$. Regarding the histologic staging of OSF, the 59 OSF specimens were further divided into moderately advanced $(n=23)$ and advanced OSF subgroups $(n=36)$ according to the histologic criteria described by Sirsat \& Pindborg (40). In this study, we did not attempt to analyze the relationship between MMO, site of involvement or histologic staging of OSF and the daily or total consumption of AQs, cigarettes and alcohol or the duration of these oral habits because our previous studies have shown no significant correlation between two of these variables $(5,6)$. However, the correlation between histologic staging of OSF and MMO or site of involvement as well as between MMO and site of involvement was analyzed for statistical significance by chi-square test. Furthermore, the correlation between the mean values of $I_{380 \pm 15 \mathrm{~nm}}, I_{460 \pm 15 \mathrm{~nm}}$, or ratio of $I_{460 \pm 15 \mathrm{~nm}} / I_{380 \pm 15 \mathrm{~nm}}$ and OSF patients' age, MMO, site of involvement, histologic staging of OSF or oral habits was analyzed for statistical significance by Mann-Whitney rank sum test. A $P$-value of less than 0.05 was considered to be statistically significant.

\section{Results}

In this study, at 330-nm excitation all the fluorescence spectra of NOM, OSF, FHOSF, and OLOSF had two significant emission peaks: one at $380 \mathrm{~nm}$ and the other at $460 \mathrm{~nm}$ (Fig. 1). The spectrum of the OSF mucosa had a significantly higher 380-nm emission peak and a significantly lower 460-nm emission peak than the spectra of NOM, FHOSF, and OLOSF samples. However, there was no significant difference in the pattern and intensities of 380- and 460-nm emission peaks between NOM and FHOSF or OLOSF samples as well as between FHOSF and OLOSF samples (Fig. 1).

When the mean $( \pm S D)$ fluorescence intensities at $380 \pm 15 \mathrm{~nm}$ $\left(I_{380 \pm 15 \mathrm{~nm}}\right)$ and $460 \pm 15 \mathrm{~nm}\left(I_{460 \pm 15 \mathrm{~nm}}\right)$ emission peaks and the mean ratio of $I_{460 \pm 15 \mathrm{~nm}} / I_{380 \pm 15 \mathrm{~nm}}$ were compared between groups, we found that OSF group had a significantly higher mean value of $I_{380 \pm 15} \mathrm{~nm}$, a significantly lower mean value of $I_{460 \pm 15 \mathrm{~nm}}$, and a significantly lower mean ratio of $I_{460 \pm 15 \mathrm{~nm}} / I_{380 \pm 15 \mathrm{~nm}}$ than the NOM, FHOSF, and OLOSF groups $(P<0.001)$. However, no significant differences in the mean values of $I_{380 \pm 15 \mathrm{~nm}}, I_{460 \pm 15 \mathrm{~nm}}$,

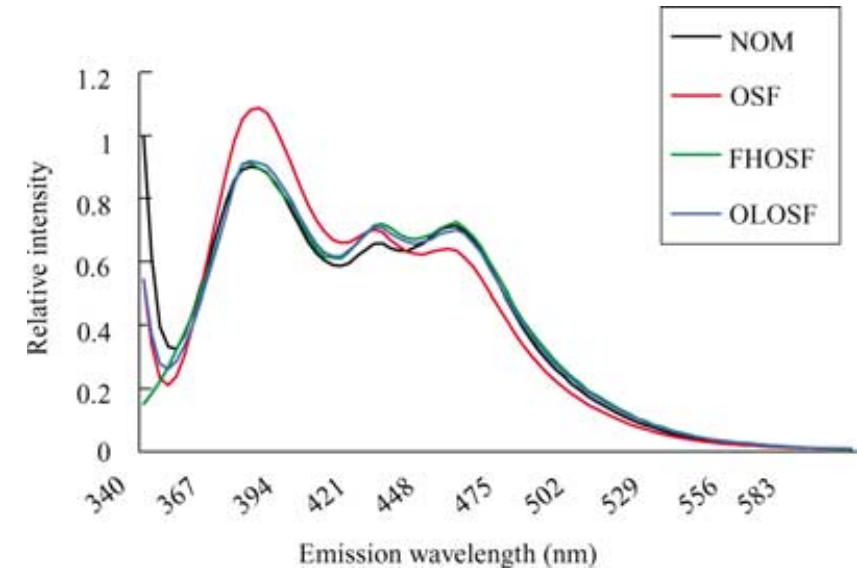

Fig. 1. Mean auto-fluorescence spectra of samples of normal oral mucosa (NOM), oral submucous fibrosis mucosa (OSF), friction hyperkeratosis on OSF buccal mucosa (FHOSF), and oral leukoplakia on OSF buccal mucosa (OLOSF) at 330-nm excitation.

and ratio of $I_{460 \pm 15 \mathrm{~nm}} / I_{380 \pm 15 \mathrm{~nm}}$ were found between NOM and FHOSF or OLOSF samples as well as between FHOSF and OLOSF samples $(P>0.05$, Table 1$)$.

The mean $( \pm \mathrm{SD})$ MMO of 59 OSF patients was $31.1 \pm$ $10.9 \mathrm{~mm}$; 30 had $\mathrm{MMO} \leq 29 \mathrm{~mm}$; and $29 \mathrm{had} \mathrm{MMO}>29 \mathrm{~mm}$. The soft palate, retromolar area, and buccal mucosa were the three sites that were involved by OSF in every case, with extra involvement of labial mucosa in 40 cases (67.8\%), of floor of the mouth in 31 cases (52.5\%), and of tongue in 33 cases (55.9\%). Of 59 OSF cases, the disease involved three sites in 15 cases, four sites in 10 cases, five sites in eight cases, and six sites in 26 cases. Chisquare test revealed that there was a significant correlation between MMO and site of involvement $(P<0.001)$. However, no significant correlation was found between histologic staging of OSF and MMO or site of involvement ( $P>0.05$, data not shown).

When OSF patients were divided into different subgroups according to the age, MMO, site of involvement, and histologic staging of OSF, there were no significant differences in the mean

Table 1. Average fluorescence intensities at $380 \pm 15 \mathrm{~nm}\left(I_{380 \pm 15 \mathrm{~nm}}\right)$ and $460 \pm 15 \mathrm{~nm}\left(I_{460 \pm 15 \mathrm{~nm}}\right)$ emission peaks and the mean ratio of $I_{460 \pm 15 \mathrm{~nm}} / I_{380 \pm 15 \mathrm{~nm}}$ for normal oral mucosa (NOM), oral submucous fibrosis mucosa (OSF), friction hyperkeratosis on OSF buccal mucosa (FHOSF), and oral leukoplakia on OSF buccal mucosa (OLOSF)

\begin{tabular}{llll}
\hline Groups & $\begin{array}{l}I_{380 \pm 15 \mathrm{~nm}} \\
(\text { mean } \pm \text { SD })\end{array}$ & $\begin{array}{l}I_{460 \pm 15 \mathrm{~nm}} \\
(\text { mean } \pm \text { SD })\end{array}$ & $\begin{array}{l}I_{460 \pm 15 \mathrm{~nm}} / I_{380 \pm 15 \mathrm{~nm}} \\
(\operatorname{mean} \pm \mathrm{SD})\end{array}$ \\
\hline NOM $(n=15)$ & $0.82 \pm 0.04$ & $0.62 \pm 0.13$ & $0.76 \pm 0.07$ \\
OSF $(n=59)$ & $0.97 \pm 0.13^{*}$ & $0.55 \pm 0.07^{*}$ & $0.59 \pm 0.15^{*}$ \\
FHOSF $(n=5)$ & $0.82 \pm 0.14$ & $0.64 \pm 0.07$ & $0.80 \pm 0.19$ \\
OLOSF $(n=29)$ & $0.83 \pm 0.13$ & $0.62 \pm 0.08$ & $0.77 \pm 0.22$ \\
\hline
\end{tabular}

*Significant differences were found between OSF and NOM, FHOSF or OLOSF samples by Mann-Whitney rank sum test with $P<0.001$. 
Table 2. Correlation between mean fluorescence intensities at $380 \pm 15 \mathrm{~nm}\left(I_{380 \pm 15 \mathrm{~nm}}\right)$ and $460 \pm 15 \mathrm{~nm}\left(I_{460 \pm 15 \mathrm{~nm}}\right)$ emission peaks or the mean ratio of $I_{460 \pm 15 \mathrm{~nm}} / I_{380 \pm 15 \mathrm{~nm}}$ and OSF patients' age, maximum mouth opening (MMO), site of involvement, or histologic staging of OSF

\begin{tabular}{|c|c|c|c|}
\hline Parameters & $\begin{array}{l}I_{380 \pm 15 \mathrm{~nm}} \\
(\text { mean } \pm S D)\end{array}$ & $\begin{array}{l}I_{460 \pm 15 \mathrm{~nm}} \\
(\text { mean } \pm S D)\end{array}$ & $\begin{array}{l}I_{460 \pm 15 \mathrm{~nm}} / I_{380 \pm 15 \mathrm{~nm}} \\
(\mathrm{mean} \pm \mathrm{SD})\end{array}$ \\
\hline \multicolumn{4}{|l|}{ Age (yeas) } \\
\hline$\leq 38(n=30)$ & $0.98 \pm 0.12$ & $0.55 \pm 0.07$ & $0.57 \pm 0.14$ \\
\hline$>38(n=29)$ & $0.95 \pm 0.14$ & $0.55 \pm 0.07$ & $0.60 \pm 0.15$ \\
\hline \multicolumn{4}{|l|}{ MMO (mm) } \\
\hline$\leq 29(n=30)$ & $0.95 \pm 0.11$ & $0.56 \pm 0.07$ & $0.61 \pm 0.14$ \\
\hline$>29(n=29)$ & $0.99 \pm 0.14$ & $0.54 \pm 0.08$ & $0.57 \pm 0.15$ \\
\hline \multicolumn{4}{|l|}{ Site of involvement } \\
\hline 3 or 4 sites $(n=25)$ & $0.97 \pm 0.13$ & $0.54 \pm 0.06$ & $0.57 \pm 0.14$ \\
\hline 5 or 6 sites $(n=34)$ & $0.96 \pm 0.13$ & $0.55 \pm 0.08$ & $0.59 \pm 0.15$ \\
\hline \multicolumn{4}{|l|}{ Histologic staging of OSF } \\
\hline $\begin{array}{l}\text { Moderately advanced } \\
(n=23)\end{array}$ & $0.97 \pm 0.15$ & $0.55 \pm 0.07$ & $0.59 \pm 0.16$ \\
\hline Advanced $(n=36)$ & $0.97 \pm 0.11$ & $0.55 \pm 0.07$ & $0.59 \pm 0.14$ \\
\hline
\end{tabular}

No significant differences are found between the mean value of $I_{380 \pm 15 \mathrm{~nm}}$, $I_{460 \pm 15 \mathrm{~nm}}$, or ratio of $I_{460 \pm 15 \mathrm{~nm}} / I_{380 \pm 15 \mathrm{~nm}}$ and OSF patients' age, MMO, site of involvement, or histologic staging of OSF by Mann-Whitney rank sum test.

Table 3. Correlation between OSF patients' oral habits and the mean fluorescence intensities at $380 \pm 15 \mathrm{~nm}\left(I_{380 \pm 15 n m}\right)$ and $460 \pm 15 \mathrm{~nm}$ $\left(I_{460 \pm 15 \mathrm{~nm}}\right)$ emission peaks or the mean ratio of $I_{460 \pm 15 \mathrm{~nm}} / I_{380 \pm 15 \mathrm{~nm}}$

\begin{tabular}{|c|c|c|c|}
\hline OSF patients' oral habits & $\begin{array}{l}I_{380 \pm 15 \mathrm{~nm}} \\
(\text { mean } \pm S D)\end{array}$ & $\begin{array}{l}I_{460 \pm 15 \mathrm{~nm}} \\
(\text { mean } \pm S D)\end{array}$ & $\begin{array}{l}I_{460 \pm 15 \mathrm{~nm}} / I_{380 \pm 15 \mathrm{~nm}} \\
(\mathrm{mean} \pm \mathrm{SD})\end{array}$ \\
\hline \multicolumn{4}{|c|}{ Daily areca quid consumption } \\
\hline$\leq 20$ quids $(n=33)$ & $0.98 \pm 0.13$ & $0.54 \pm 0.07$ & $0.57 \pm 0.15$ \\
\hline$>20$ quids $(n=26)$ & $0.95 \pm 0.12$ & $0.56 \pm 0.07$ & $0.61 \pm 0.14$ \\
\hline \multicolumn{4}{|c|}{ Duration of chewing areca quids } \\
\hline$\leq 12$ years $(n=31)$ & $0.96 \pm 0.13$ & $0.55 \pm 0.07$ & $0.59 \pm 0.15$ \\
\hline$>12$ years $(n=28)$ & $0.97 \pm 0.12$ & $0.55 \pm 0.07$ & $0.58 \pm 0.15$ \\
\hline \multicolumn{4}{|c|}{ Total consumption of areca quids } \\
\hline$\leq 73000$ quids $(n=30)$ & $1.00 \pm 0.12$ & $0.53 \pm 0.07$ & $0.54 \pm 0.14$ \\
\hline$>73000$ quids $(n=29)$ & $0.93 \pm 0.13$ & $0.57 \pm 0.07$ & $0.63 \pm 0.14$ \\
\hline \multicolumn{4}{|l|}{ Daily cigarette consumption } \\
\hline$\leq 1$ package $(n=47)$ & $0.98 \pm 0.13$ & $0.55 \pm 0.07$ & $0.58 \pm 0.14$ \\
\hline$>1$ package $(n=12)$ & $0.94 \pm 0.14$ & $0.55 \pm 0.08$ & $0.61 \pm 0.17$ \\
\hline \multicolumn{4}{|c|}{ Duration of smoking cigarettes } \\
\hline$\leq 16$ years $(n=31)$ & $1.00 \pm 0.12$ & $0.53 \pm 0.07$ & $0.54 \pm 0.14$ \\
\hline$>16$ years $(n=28)$ & $0.93 \pm 0.12$ & $0.57 \pm 0.06$ & $0.63 \pm 0.14$ \\
\hline \multicolumn{4}{|c|}{ Total consumption of cigarettes } \\
\hline $\begin{array}{l}\leq 109500 \text { cigarettes } \\
(n=30)\end{array}$ & $1.01 \pm 0.12$ & $0.53 \pm 0.07$ & $0.54 \pm 0.14$ \\
\hline $\begin{array}{l}>109500 \text { cigarettes } \\
(n=29)\end{array}$ & $0.92 \pm 0.12$ & $0.57 \pm 0.07$ & $0.63 \pm 0.14$ \\
\hline \multicolumn{4}{|l|}{ Weekly alcohol consumption } \\
\hline$\leq 63 \mathrm{~g}(n=31)$ & $0.99 \pm 0.14$ & $0.54 \pm 0.08$ & $0.56 \pm 0.16$ \\
\hline$>63 \mathrm{~g}(n=28)$ & $0.94 \pm 0.11$ & $0.56 \pm 0.06$ & $0.61 \pm 0.13$ \\
\hline \multicolumn{4}{|l|}{ Duration of alcohol drinking } \\
\hline$\leq 10$ years $(n=32)$ & $1.01 \pm 0.13$ & $0.54 \pm 0.07$ & $0.55 \pm 0.15$ \\
\hline$>10$ years $(n=27)$ & $0.92 \pm 0.11$ & $0.57 \pm 0.07$ & $0.63 \pm 0.14$ \\
\hline \multicolumn{4}{|l|}{ Total consumption of alcohol } \\
\hline$\leq 39208 \mathrm{~g}(n=30)$ & $1.00 \pm 0.13$ & $0.53 \pm 0.08$ & $0.56 \pm 0.16$ \\
\hline$>39208 \mathrm{~g}(n=29)$ & $0.94 \pm 0.11$ & $0.56 \pm 0.06$ & $0.62 \pm 0.13$ \\
\hline
\end{tabular}

No significant correlation was found between the OSF patients' oral habits and the mean value of $I_{380 \pm 15 \mathrm{~nm}}, I_{460 \pm 15 \mathrm{~nm}}$, or ratio of $I_{460 \pm 15 \mathrm{~nm}} / I_{380 \pm 15 \mathrm{~nm}}$ by MannWhitney rank sum test. values of $I_{380 \pm 15 \mathrm{~nm}}, I_{460 \pm 15 \mathrm{~nm}}$, and ratio of $I_{460 \pm 15 \mathrm{~nm}} / I_{380 \pm 15 \mathrm{~nm}}$ between any two of the subgroups $(P>0.05$, Table 2$)$. In addition, no significant differences were found between the mean values of $I_{380 \pm 15 \mathrm{~nm}}, I_{460 \pm 15 \mathrm{~nm}}$, or ratio of $I_{460 \pm 15 \mathrm{~nm}} / I_{380 \pm 15 \mathrm{~nm}}$ and the OSF patients' oral habits including the daily/weekly consumption of AQs, cigarettes and alcohol and duration of these oral habits $(P>0.05$, Table 3$)$.

\section{Discussion}

In this study, we showed that at 330-nm excitation the spectra of OSF mucosal samples have a significantly higher 380-nm emission peak and a significantly lower 460-nm emission peak than the spectra of NOM, FHOSF, and OLOSF samples. In addition, OSF mucosal samples had a significantly higher mean value of $I_{380 \pm 15 \mathrm{~nm}}$, a significantly lower mean value of $I_{460 \pm 15 \mathrm{~nm}}$, and a significantly lower mean ratio of $I_{460 \pm 15 \mathrm{~nm}} / I_{380 \pm 15 \mathrm{~nm}}$ than the NOM, FHOSF, and OLOSF samples $(P<0.001)$. Our previous study also found that at 330-nm excitation oral cancer tissues have a significantly lower 380-nm emission peak and a significantly higher 460-nm emission peak than the NOM tissues (35). These findings indicate that OSF mucosa has a very unique pattern of auto-fluorescence spectrum that can be used for in vivo real-time diagnosis of OSF. However, this study showed no significant difference in the pattern and peak intensity of the spectrum between moderately advanced and advanced OSF. This suggests that although auto-fluorescence spectroscopy can detect increased amount of collagen in the subepithelial connective tissue, it is not sensitive enough to differentiate the histologic changes between the moderately advanced and advanced OSF.

Although the pathogenesis of OSF is still unclear, previous studies have suggested that the occurrence of OSF may be due to increased production of collagen and decreased degradation of collagen in the subepithelial connective tissue of the oral mucosa (7-15). Areca nut alkaloid as well as cytokines and growth factors secreted by the mononuclear inflammatory cells in the OSF mucosa can stimulate fibroblast proliferation and upregulate collagen synthesis $(7,8)$. Areca nut polyphenols and lysyl oxidase produced by the OSF fibroblasts can stabilize the collagen structures that become resistant to degradation by collagenase $(9,10$, 12, 13). In addition, the more stable collagen type I trimer produced by OSF fibroblasts is also resistant to degradation by collagenase (11). All of these factors contributed to the deposition of excessive amount of collagen in the subepithelial connective 
tissue of the OSF mucosa. Previous studies have shown that at 330-nm excitation, the 380- and 460-nm emission peaks of the auto-fluorescence spectra for oral mucosal tissues reflect the collagen content in the subepithelial connective tissue and the $\mathrm{NADH}$ content in the epithelial cells, respectively $(25,26,35,38$, 39). Therefore, the increased collagen content in subepithelial connective tissue could explain why OSF mucosa had a significantly higher intensity of the 380-nm emission peak of the autofluorescence spectra than the NOM samples. Furthermore, compared to normal oral epithelium the markedly atrophic OSF epithelium allowed more excitation energy to penetrate into the subepithelial connective tissue and more collagen-derived emission fluorescence to go through. This factor also gave some contribution to the high intensity of the 380-nm emission peak of the auto-fluorescence spectra of OSF samples. In addition, the markedly atrophic OSF epithelium might contain less amount of $\mathrm{NADH}$ than the normal oral epithelium. The fibrosis-induced reduction of blood vessels in the lamina propria might also diminish the metabolic rate of oral epithelial cells, which in turn resulted in a lower NADH content in the OSF epithelium. Therefore, OSF mucosa had a significantly lower intensity of the 460-nm emission peak than the NOM samples.

In this study, no significant differences in the pattern and intensity of the 380- and 460-nm emission peaks were found between NOM and FHOSF or OLOSF samples. FH and OL on the OSF mucosa were supposed to have a higher 380-nm emission peak of auto-fluorescence spectra at 330-nm excitation than the NOM samples because of the increased collagen content in the subepithelial connective tissue of OSF mucosa. However, the thickened hyperkeratotic and hyperplastic epithelium of FHOSF and OLOSF lesions might reduce the intensity of both excitation energy and collagen-derived emission fluorescence, resulting in a decreased 380-nm emission peak that was about equal to that of the NOM samples. In addition, the thickened and hyperplastic epithelium of FHOSF and OLOSF lesions were supposed to contain more $\mathrm{NADH}$ than the normal oral epithelium and in turn have a higher 460-nm emission peak than the NOM samples. However, the fibrosis-induced decrease of blood vessels in the lamina propria might also reduce the metabolic rate and $\mathrm{NADH}$ content of the hyperplastic epithelial cells, resulting in an attenuated 460$\mathrm{nm}$ emission peak that was about equal to that of the NOM samples. Our findings suggest that fibrosis in the lamina propria and submucosa of the oral mucosa underneath the oral epithelial lesions like FH and OL may alter the auto-fluorescence spectral pattern and intensity of the emission peaks. Therefore, the autofluorescence measurements obtained from oral lesions with underlying fibrosis should be explained with special attention.
In this study, chi-square test revealed that there was a significant correlation between MMO and site of involvement $(P<0.001)$. This suggests that both indices may be symbolic of the development and clinical severity of OSF. Our recent study also found that MMO was the most important symptom for an early stage OSF patient (MMO $\geq 35 \mathrm{~mm}$ ) to be differentiated from advanced OSF patients (MMO $<35 \mathrm{~mm}$ ) (41). Our data did not show an association between histologic staging of OSF and MMO or site of involvement. In addition, no significant correlations were found between the mean values of $I_{380 \pm 15 \mathrm{~nm}}, I_{460 \pm 15 \mathrm{~nm}}$, or ratio of $I_{460 \pm 15 \mathrm{~nm}} / I_{380 \pm 15 \mathrm{~nm}}$ and the OSF patients' age, MMO, site of involvement, oral habits or histologic staging of OSF. No significant association between these variables could be due to the complexity of OSF and involvement of multiple factors in the pathogenesis of OSF.

To the best of our knowledge, this paper was the first report describing the characteristic auto-fluorescence spectral pattern of OSF lesions. We found that at 330-nm excitation the spectrum of the OSF mucosa had a significantly higher 380-nm emission peak and a significantly lower 460-nm emission peak than the spectra of NOM, FHOSF, and OLOSF samples. Furthermore, OSF samples had a significantly higher mean value of $I_{380 \pm 15} \mathrm{~nm}$, a significantly lower mean value of $I_{460 \pm 15 \mathrm{~nm}}$, and a significantly lower mean ratio of $I_{460 \pm 15 \mathrm{~nm}} / I_{380 \pm 15 \mathrm{~nm}}$ than the NOM, FHOSF, and OLOSF samples $(P<0.001)$. Because OSF mucosa has a very unique pattern of auto-fluorescence spectrum, we conclude that auto-fluorescence spectroscopy is a good method for real-time diagnosis of OSF.

\section{References}

1. Maher R, Lee AJ, Warnakulasuriya KAAS, Lewis JA, Johnson NW. Role of areca nut in the causation of oral submucous fibrosis: a casecontrol study in Pakistan. J Oral Pathol Med 1994; 23: 65-9.

2. Murti PR, Bhonsle RB, Gupta PC, Daftary DK, Pindborg JJ, Mehta FS. Etiology of oral submucous fibrosis with special reference to the role of areca nut chewing. J Oral Pathol Med 1995; 24: 145-52.

3. Shah N, Sharma PP. Role of chewing and smoking habits in the etiology of oral submucous fibrosis (OSF): a case-control study. $J$ Oral Pathol Med 1998; 27: 475-9.

4. Chiang CP, Lang MJ, Liu BY, et al. Expression of proliferating cell nuclear antigen (PCNA) in oral submucous fibrosis, oral epithelial hyperkeratosis and oral epithelial dysplasia in Taiwan. Oral Oncol 2000; 36: 353-9.

5. Chiang $\mathrm{CP}$, Wu HY, Liu BY, Wang JT, Kuo MYP. Quantitative analysis of immunocompetent cells in oral submucous fibrosis in Taiwan. Oral Oncol 2002; 38: 52-63.

6. Chiang CP, Hsieh RP, Chen THH, et al. High incidence of autoantibodies in Taiwanese patients with oral submucous fibrosis. J Oral Pathol Med., 2002; 31: 402-9. 
7. Harvey W, Scutt A, Meghji S, Canniff JP. Stimulation of human buccal mucosa fibroblasts in vitro by betel-nut alkaloids. Arch Oral Biol 1986; 31: 45-9.

8. Haque MF, Harris M, Meghji S, Barrett AW. Immunolocalization of cytokines and growth factors in oral submucous fibrosis. Cytokine 1998; 10: 713-9.

9. Kuttan R, Donnely PV, Di Ferrante N. Collagen treated with $(+)-$ catechin becomes resistant to the action of mammalian collagenase. Experientia 1981; 37: 221-3.

10. Scutt A, Meghji S, Canniff JP, Harvey W. Stabilisation of collagen by betel nut polyphenols as a mechanism in oral submucous fibrosis. Experientia 1987; 43: 391-3.

11. Kuo MYP, Chen HM, Hahn LJ, Hsieh CC, Chiang CP. Collagen biosynthesis in human oral submucous fibrosis fibroblast cultures. $J$ Dent Res 1995; 74: 1783-8.

12. Ma RH, Tsai CC, Shieh TY. Increased lysyl oxidase activity in fibroblasts cultured from oral submucous fibrosis associated with betel nut chewing in Taiwan. J Oral Pathol Med 1995; 24: 407-12.

13. Trivedy C, Warnakulasuriya KAAS, Hazarey VK, Tavassoli M, Sommer P, Johnson NW. The upregulation of lysyl oxidase in oral submucous fibrosis and squamous cell carcinoma. J Oral Pathol Med 1999; 28: 246-51.

14. Shieh TY, Yang JF. Collagenase activity in oral submucous fibrosis. Proc Natl Sci Counc ROC (B) 1992; 16: 106-10.

15. Tsai CC, Ma RH, Shieh TY. Deficiency in collagen and fibronectin phagocytosis by human buccal mucosa fibroblasts in vitro as a possible mechanism for oral submucous fibrosis. J Oral Pathol Med 1999; 28: 59-63.

16. Ko YC, Chiang TA, Chang SJ, Hsieh SF. Prevalence of betel quid chewing habit in Taiwan and related sociodemographic factors. $J$ Oral Pathol Med 1992; 21: 261-4.

17. Pindborg JJ, Mehta FS, Gupta PC, Daftary DK. Prevalence of oral submucous fibrosis among 50915 Indian villagers. Br J Cancer 1968; 22: $646-54$.

18. Lay KM, Sein K, Myint A, Ko SK, Pindborg JJ. Epidemiolgic study of 600 villagers of oral precancerous lesions in Bilugyun: preliminary report. Community Dent Oral Epidemiol 1982; 10: 152-5.

19. Seedat HA. Oral submucous fibrosis in Durban, Natal: a study of its epidemiology, aetiology and morphological features. Thesis. Stellenbosch, South Africa: University of Stellenbosch, 1985.

20. Zain RB, Ikeda N, Razak IA, et al. A national epidemiological survey of oral mucosal lesions in Malaysia. Community Dent Oral Epidemiol 1997; 25: 377-83.

21. Tang JG, Jian XF, Gao ML, Ling TY, Zhang KH. Epidemiological survey of oral submucous fibrosis in Xiangtan City, Hunan province, China. Community Dent Oral Epidemiol 1997; 25: 177-80.

22. Tang GC, Pradhan A, Alfano RR. Spectroscopic differences between human cancer and normal lung and breast tissues. Lasers Surg Med 1989; 9: 290-5.

23. Hung J, Lam S, LeRiche JC, Palcic B. Autofluorescence of normal and malignant bronchial tissue. Lasers Surg Med 1991; 11: 99-105.

24. Mahadevan A, Mitchell MF, Silva E, Thomsen S, Richard-Kortum RR. A study of the fluorescence properties of normal and neoplastic human cervical tissue. Lasers Surg Med 1993; 13: 647-55.

25. Ramanujam N, Mitchell MF, Mahadevan A, et al. In vivo diagnosis of cervical intraepithelial neoplasia using 337-nm-excited laser-induced fluorescence. Proc Natl Acad Sci USA 1994; 91: 10193-7.
26. Bottiroli G, Corce AC, Locatelli D, et al. Natural fluorescence of normal and neoplastic human colon: a comprehensive 'ex vivo' study. Lasers Surg Med 1995; 16: 48-60.

27. Romer TJ, Fitzmaurice M, Cothren-Kortum R, Petras R, Sivak MV, Kramer LR. Laser-induced fluorescence microscopy of normal colon and dysplasia in colonic adenomas: implication for spectroscopic diagnosis. Am J Gastroenterol 1995; 90: 81-7.

28. Wang CY, Lin JK, Chen BF, Chiang HK. Autofluorescence spectroscopic differentiation between normal and cancerous colorectal tissues by means of a two-peak ratio algorithm. J Formos Med Assoc 1999; 98: 837-43.

29. Koenig F, McGovern FJ, Enquist H, Larne R, Deutsch TF, Schomacker KT. Autofluorescence guided biopsy for the early diagnosis of bladder carcinoma. J Urol 1998; 159: 1871-5.

30. Betz CS, Mehlmann M, Rick K, et al. Autofluorescence imaging and spectroscopy of normal and malignant mucosa in patients with head and neck cancer. Lasers Surg Med 1999; 25: 323-34.

31. Schantz SP, Kolli V, Savage $\mathrm{HE}$, et al. In vivo native cellular fluorescence and histological characteristics of head and neck cancer. Clin Cancer Res 1998; 4: 1177-82.

32. Eker C, Ryland R, Svanberg K, Andersson-Engles S. Multivariate analysis of laryngeal fluorescence spectra recorded in vivo. Lasers Surg Med 2001; 28: 259-66.

33. Gillenwater A, Jacob R, Ganeshappa R, et al. Noninvasive diagnosis of oral neoplasia based on fluorescence spectroscopy and native tissue autofluorescence. Arch Otolaryngol Head Neck Surg 1998; 124: $1251-8$.

34. Ingrams DR, Dhingra JK, Roy K, et al. Autofluorescence characteristics of oral mucosa. Head Neck 1997; 19: 27-32.

35. Chen CT, Chiang HK, Chow SN, et al. Autofluorescence in normal and malignant human oral tissues and in DMBA-induced hamster buccal pouch carcinogenesis. J Oral Pathol Med 1998; 27: $470-4$.

36. Udenfriend S (ed.). Fluorescence Assay in Biology and Medicine, Vol. 2. New York: Academic Press, 1969.

37. Fasman GD (ed.). Handbook of Biochemistry and Molecular Biology, 3rd edn. Cleveland: CRC Press, 1975; 205-10.

38. Alfano RR, Das BB, Cleary J, Prudent R, Celmer E. Light sheds light on cancer-distinguishing malignant tumors from benign tissues and tumors. Bul NY Acad Med 1991; 67: 143-50.

39. Glasgold R, Glasgold M, Savage H, Pinto J, Alfano R, Schantz S. Tissue autofluorescence as an intermediate endpoint in NMBAinduced esophageal carcinogenesis. Cancer Lett 1994; 82: 33-41.

40. Sirsat SM, Pindborg JJ. Subepithelial changes in oral submucous fibrosis. Acta Pathol Microbiol Scand J 1967; 70: 161-73.

41. Chiu CJ, Lee WC, Chiang CP, Hahn LJ, Kuo YS, Chen CJ. A scoring system for the early detection of oral submucous fibrosis based on a self-administered questionnaire. J Public Health Dent 2002; 62: $28-31$.

\section{Acknowledgements}

This study was supported by research grants of NSC90-2736-L-002-002 and NSC90-2736-L-002-003 from the National Science Council, Taipei, Taiwan. 